\title{
WILEY-VCH
}

\section{Why are Hot Holes Easier to Extract than Hot Electrons from Methylammonium Lead Iodide Perovskite?}

Ibrahim Dursun, ${ }^{1,2,+}$ Partha Maity, ${ }^{1,2, \dagger}$ Jun Yin $^{2}$, Bekir Turedi, ${ }^{1,2}$ Ayan A. Zhumekenov, ${ }^{1,2}$ Kwang Jae Lee, ${ }^{1,2}$ Omar F. Mohammed, ${ }^{2, *}$ and Osman M. Bakr ${ }^{1,2, *}$

I. Dursun, Dr. P. Maity, Dr. J. Yin, B. Turedi, A. A. Zhumekanov, Dr. K. J. Lee, Prof. O. F. Mohammed, Prof. O. M. Bakr

Division of Physical Science and Engineering (PSE), King Abdullah University of Science and Technology (KAUST), Thuwal 23955, Kingdom of Saudi Arabia

E-mail: omar.abdelsaboor@kaust.edu.sa

E-mail: osman.bakr@kaust.edu.sa

I. Dursun, B. Turedi, A. A. Zhumekanov, Dr. K. J. Lee, Prof. O. M. Bakr

KAUST Catalysis Center (KCC), Division of Physical Science and Engineering (PSE), King Abdullah University of Science and Technology (KAUST), Thuwal 23955, Kingdom of Saudi Arabia

E-mail: osman.bakr@kaust.edu.sa

Keywords: Halide perovskite, hot carriers, carrier temperature, carrier extraction, density functional theory

Charge-carriers photoexcited above a semiconductor's bandgap rapidly thermalize to the bandedge. The cooling of these difficult to collect 'hot' carriers caps the available photon energy that solar cells - including efficient perovskite solar cells - may utilize. Here, the dynamics and efficiency of hot carrier extraction from $\mathrm{MAPbI}_{3}(\mathrm{MA}=$ methylammonium) perovskite by SpiroOMeTAD (a hole-transporting layer) and $\mathrm{TiO}_{2}$ (an electron-transporting layer) are investigated and explained using both ultrafast electronic spectroscopy and theoretical modelling. Timeresolved spectroscopy reveals a quasi-equilibrium distribution of hot carriers forming upon excess-energy excitation of the perovskite - a distribution largely unaffected by the presence of $\mathrm{TiO}_{2}$. In contrast, the quasi-equilibrium distribution of hot carriers is virtually nonexistent when Spiro-OMeTAD is present, which is indicative of efficient hot hole extraction at the interface of $\mathrm{MAPbI}_{3}$. Density functional theory (DFT) calculations predict that deep energy-levels of $\mathrm{MAPbI}_{3}$ exhibit electronically delocalized character, with significant overlap with the localized valence band charge of the Spiro-OMeTAD molecules lying on the surface of MAPbI 3 . Consequently, hot holes are easily extracted from the deep energy-levels of $\mathrm{MAPbI}_{3}$ by SpiroOMeTAD. These findings uncover the origins of efficient hot hole extraction in perovskites 


\section{WILEY-VCH}

and offer a practical blue print for optimizing solar cell interlayers to enable hot carrier utilization.

\section{Introduction}

Renewable energy from solar cells has reached cost parity with fossil fuels in many parts of the world, ${ }^{[1]}$ driven largely by cost reductions achieved by mass manufacturing photovoltaic devices containing a single junction (i.e., one absorbing semiconductor). ${ }^{[2]}$ While these devices are amenable to mass manufacturing due to their relatively simple architecture, they are also limited in the solar spectrum that they can exploit, which is set by the bandgap energy of their absorber. Photons that exceed the bandgap's energy excite the charge-carriers to energy levels above the bandgap, where upon the carriers quickly relax back (i.e., cooldown) to the bandgap edge, essentially dissipating all energy in excess of the bandgap. This innate process of rapid hot carrier cooling, preceding the carrier recombination at the bandgap's edge, is a major source of loss in semiconductor materials that limits their photovoltaic performance. ${ }^{[3]}$ In single junction solar cells such losses set an efficiency limit of 33\% - known as the Shockley-Queisser (SQ) limit ${ }^{[3 b]}-$ on the harvestable solar energy. Potentially pushing the solar cell efficiencies beyond this limit entails overcoming the narrow time window of carrier cooling ( $\sim$ hundreds of femtoseconds, fs) and addressing the energy-level alignment mismatch preventing the effective extraction of hot carriers from the absorbing semiconductor.

Recently, solar cells based on a low-temperature solution-processable class of absorbing semiconductors, known as metal halide perovskites, have been the subject of intense investigation. The initial interest in these materials stemmed from the impressive efficiencies that their solar cells could achieve, despite their simple processing routes, offering a prospect for a cheap yet efficient photovoltaic technology (current power conversion efficiency ca. $23 \%) .{ }^{[4]}$ However, the scope of halide perovskite optoelectronics has expanded to encompass photodetectors, ${ }^{[5]}$ scintillators, ${ }^{[6]}$ light emitting diodes, ${ }^{[7]}$ and lasers. ${ }^{[8]}$ Understanding the cooling dynamics of hot carriers is of relevance to all these applications, and utilizing (the 


\section{WILEY-VCH}

currently unutilized) hot carriers is potentially beneficial (varying extent) to all the aforementioned optoelectronic technologies.

The study of hot carrier dynamics in methylammonium lead iodide $\left(\mathrm{CH}_{3} \mathrm{NH}_{3} \mathrm{PbI}_{3}\right.$, or $\mathrm{MAPbI}_{3}$ ) - an archetypal perovskite solar cell material - has received significant attention over the last several years. ${ }^{[9]}$ With a few studies uncovering qualitatively different cooling behavior exhibited by perovskites in comparison to other conventional semiconductor materials used in solar cells. ${ }^{[10]}$ However, the extraction of these hot carriers before they quickly relax to the band edges still remains largely elusive for the $\mathrm{MAPbI}_{3}$ perovskite films; although there are few works about the extraction from other perovskites systems, including $\mathrm{MAPbBr}_{3},{ }^{[11]} \mathrm{CsPbI}_{3},{ }^{[12]}$ and $\mathrm{CsPbBr}_{3}{ }^{[13]}$ perovskite nanocrystals.

In this report, using a combination of femtosecond transient absorption (fs-TA) spectroscopy and density functional theory (DFT) modelling, we explore and decipher the mechanisms of hot carrier relaxation and extraction at the interface between $\mathrm{MAPbI}_{3}$ perovskite and $\mathrm{TiO}_{2}$ electron-transporting layer (ETL) or Spiro-OMeTAD hole-transporting layer (HTL) - the commonly used carrier-selective contacts in perovskite solar cells. In contrast to $\mathrm{TiO}_{2}$, the presence of Spiro-OMeTAD results in the absence of spectral broadening of the hot carrier distribution, which is a spectroscopic signature indicative of efficient hot hole extraction from pristine perovskite to the HTL. Our modelling indicates that the efficient extraction of hot holes is driven mainly by the strong overlap of the localized charge of the valence band from SpiroOMeTAD with aligned electronic states of delocalized character on the surface of $\mathrm{MAPbI}_{3}$.

\section{Results and Discussion}

We started exploring the hot carrier relaxation with the pristine $\mathrm{MAPbI}_{3}$ perovskite films (for preparation and characterization see Experimental Section and Figure S1, Supporting Information). Figure 1 illustrates the fs-TA spectra (see Experimental Section for details of the TA measurement setup) obtained from the $\mathrm{MAPbI}_{3}$ film following 370-nm optical excitation with an excitation pump fluence of $3 \mu \mathrm{J} / \mathrm{cm}^{2}$ (corresponding to a carrier density of $\sim 5.4 \times 10^{17}$ 


\section{WILEY-VCH}

$\mathrm{cm}^{-3}$, considering the extinction coefficient of $\mathrm{MAPbI}_{3}$ at $\left.370 \mathrm{~nm} \sim 10^{5} \mathrm{~cm}^{-1}\right) \cdot{ }^{[14]}$ The TA spectra show two characteristic features, negative $(\Delta \mathrm{A}<0)$ and positive $(\Delta \mathrm{A}>0)$ absorption change that are known as photobleaching (PB) and photoinduced absorption (PIA), respectively.

Figure 1a represents the two-dimensional (2D) pseudo-color plot of the TA spectra of spincoated $\mathrm{MAPbI}_{3}$ film on the glass substrate over a $5 \mathrm{~ns}$ time window, obtained upon excess energy excitation at $370 \mathrm{~nm}$; a fixed fluence of $3 \mu \mathrm{J} / \mathrm{cm}^{2}$ was used throughout this study unless otherwise stated. The strong photobleach signal (red color) at $750 \mathrm{~nm}$ (which is matched with the band gap $(1.6 \mathrm{eV})$ of the $\mathrm{MAPbI}_{3}$ film) originates from the band filling effect. ${ }^{[15]}$ Additionally, the broadening of the bleaching signal (green color) can be attributed to different photophysical processes such as band edge renormalization and many-body interactions. ${ }^{[16]}$ The blue color in the 2D spectrum represents the excited state absorption of either the bound or the free charge carriers (see details in a section). The normalized TA spectra in short time scale (from 210 fs to 2.9 ps after excitation) is plotted in Figure 1b, which displays a broadening of the bleach signals in the lower wavelength (high-energy) region. This broadening can be attributed to the quasi-equilibrium distribution of the hot carriers in the crystal lattice. ${ }^{[9 a, 9 b, 9 d]}$ It should be noted that charge-carriers below the valence band and above the conduction band that have energies greater than $k_{B} T$ are known as hot holes and electrons, respectively. Consequently, the temperature of such carriers is higher than the lattice temperature. Carrier temperature $\left(T_{C}\right)$ is one of the measurable parameters for quantifying the thermalization process of the hot carrier. The initial temperature ( $1500 \mathrm{~K})$ of the hot carriers drops rapidly (hundreds of fs timescale) to $\sim 600 \mathrm{~K}$ followed by a slow thermalization to the room temperature ( $300 \mathrm{~K}$ ) in picosecond timescale as demonstrated in detail in our hot carrier extraction section. In addition to that, the absorption band in the TA spectra below $600 \mathrm{~nm}$ represents the thermalization of the hot carriers to the quasi-equilibrium states at room temperature, which occurs within the temporal resolution of the laser pulses. ${ }^{[9 a]}$ The quasi-equilibrium distribution 


\section{WILEY-VCH}

(broadening of the high-energy tail) of the hot carriers is no longer observed when the measurement was performed at the band edge excitation $(720 \mathrm{~nm}$, see Figure S2, Supporting Information). Additionally, shifting of the photobleach maxima and the photoinduced absorption above $750 \mathrm{~nm}$ converts to photobleach associated with initial timescale can be due to the band-gap renormalization of the $\mathrm{MAPbI}_{3} \cdot{ }^{[15]}$ Further discussion on the thermalization and charge-carrier lifetime in $\mathrm{MAPbI}_{3}$ is summarized in Figure 1c. The gradual narrowing of the bleach dynamics (Figure 1c) towards high energy corresponds to the thermalization process of the carriers. ${ }^{[17]}$ In detail, the bleach recovery dynamics at 700 and $720 \mathrm{~nm}$ associated with single exponential formation time, while the bleach dynamics at 740 and $750 \mathrm{~nm}$ exhibit biexponential formation time. However, the recovery of all the bleach dynamics occurs biexponentially. The single exponential rise time constant varies within the temporal resolution of the instrument (<120 fs). For the bi-exponential rise, the second component varies up to $100 \mathrm{~s}$ of fs ( $540 \mathrm{fs}$ for $750 \mathrm{~nm}$ at $3 \mu \mathrm{J} / \mathrm{cm}^{2}$ ). This second component represents the intraband hot carrier cooling time. The hot carrier cooling mechanism in the $\mathrm{MAPbI}_{3}$ perovskite is associated with phonon emission. $\left.{ }^{[9 a}, 9 b, 18\right]$ Like polar inorganic semiconductors (especially for GaAs), ${ }^{[19]}$ the hot carriers in the perovskite thermalize via phonon emission, leading to a gradual decrease of the TA signal. It is worth pointing out that the relaxation of the hot carriers largely depends on the excitation conditions. The fluence dependent bleach kinetics at $750 \mathrm{~nm}$ is illustrated in Figure 1d. Although a linear increase of the PB signal with pump fluence was observed, a slow rise in ground state bleaching was also noticeable (Figure 1d inset). A slight decrease in the carrier temperature of hot carriers could also be seen (Figure S3, Supporting Information), especially at high excitation fluence and high carrier density $\left(1.44 \times 10^{18} \mathrm{~cm}^{-3}\right)$, which is attributed to the intrinsic hot phonon bottleneck effect. ${ }^{[9 b]}$ While this observation is in a good agreement with the literature ${ }^{[9 b, 9 d]}$, the contribution of the Auger heating effect to the slow cooling of the hot carriers cannot not be excluded ${ }^{[11]}$ Furthermore, we identified the nature of the photogenerated carriers in perovskite by analyzing the TA signal at near-infrared (NIR) 


\section{WILEY-VCH}

region following 370-nm excitation. The broad PIA extending up to the NIR can be due to the absorption of either free excitons or free carriers (see Figure S4a and b, Supporting Information). Low exciton binding energy ${ }^{[20]}$ and the fluence-dependent nongeminal charge recombination suggest that the broad PIA signal in the perovskite film is composed of the free carriers, not of free excitons, which agrees with previous reports. ${ }^{[21]}$ The gradual increase in the broad PIA intensity reflects the formation of free carriers from the hot carriers. In addition, we confirmed that the free carries are also generated through thermalization of the hot carriers (see Figure $\mathrm{S} 4 \mathrm{c}$, Supporting Information). ${ }^{[16]}$ The free carrier formation time $(560 \pm 35 \mathrm{fs})$, which was measured from the rise in the $850 \mathrm{~nm}$ kinetics (see Figure S4c, Supporting Information) of the $\mathrm{MAPbI}_{3}$ film.

Different photoexcitation behaviors are illustrated in Figure 2a when the perovskite is excited by three different excitations: with excess energy, at close to band edge and band edge. The excitation with excess energy can create charge-carriers in the electronically hot states. The charge recombination followed by thermalization occurs on a timescale of $>5 \mathrm{~ns}$. From the above discussion, and earlier studies ${ }^{[15,21 \mathrm{~d}]}$ we conclude that the charge-carriers in the perovskite are free, and not bound. With band-edge excitation, the recombination between free carriers (electrons and holes) provides the true carrier lifetime in the perovskite, which varies over a ns time scale. In addition to that, the thermalization of the hot carrier in $\mathrm{MAPbI}_{3}$ mainly associated with the longitudinal optical (LO) phonon emission process. Recent DFT and manybody perturbation theory calculations by Herz and co-workers have revealed that the spectral broadening of the perovskite is due to the scattering from LO phonon emission, which occurs via the Fröhlich interaction. ${ }^{[22]}$ Moreover, the coherent electron-acoustic phonon coupling was also observed during the electron transport in $\mathrm{MAPbI}_{3}$ single crystals. ${ }^{[23]}$ Finally, following the cooling of charges is the conversion to free carriers that eventually recombine to reform the ground state. The formation of the free carriers from hot exciton dissociation and subsequent electron-optical phonon coupling is observed on the sub-picosecond time scale. ${ }^{[18 b]}$ After 


\section{WILEY-VCH}

revealing the essential dynamics of hot carriers in $\mathrm{MAPbI}_{3}$, we move on to investigate the practical utilization of these carriers in photovoltaics by studying the efficiency of their extraction at the device interface. In the following section, we demonstrate the extraction of charge-carriers from $\mathrm{MAPbI}_{3}$ perovskite using an ETL and an HTL.

Figure $2 \mathrm{~b}$ depicts the steady-state PL of pristine $\mathrm{MAPbI}_{3}$ without and with the ETL, and HTL after 450-nm excitation. The PL of the perovskite is quenched in the presence of either ETL or HTL, which evidently illustrates the efficient extraction of the thermalized carriers (electrons and holes) at the interface of $\mathrm{MAPbI}_{3}$ perovskite. The impact of the charge extraction is further analyzed by comparing the time-resolved photoluminescence (TRPL) decay traces, which studied in time correlated single photon counting (TCSPC) measurement. It is worth noting that the temporal resolution of the excitation laser pulsed ( 100 ps) used in TCSPC measurement is more than that of the thermalization time of the hot carries. Therefore, the outcomes of the TRPL would reflect the extraction of the thermalized carriers. Figure $2 \mathrm{c}$ presents the TRPL decay traces monitored at $760 \mathrm{~nm}$ following $450 \mathrm{~nm}$ excitation of pristine $\mathrm{MAPbI}_{3}$ without and with ETL and HTL. The TRPL decay of the pristine perovskite shows $2.76 \pm 0.12 \mathrm{~ns}$ (see Table S1, Supporting Information) average radiative lifetime due to the nongeminate radiative recombination of the electron and hole. ${ }^{[21 \mathrm{~d}, 24]}$ Moreover, the radiative lifetime of the $\mathrm{MAPbI}_{3}$ can varies from sub-ns to hundreds of ns depending on the crystallite dimension (grain size) of the $\mathrm{MAPbI}_{3}$ and fluence of the excitation source. ${ }^{[25]}$ Interestingly, the average radiative lifetime of the $\mathrm{MAPbI}_{3}$ becomes very short in the presence of ETL $(0.68 \pm$ $0.04 \mathrm{~ns})$ as well as HTL $(0.76 \pm 0.04 \mathrm{~ns})$ (see Table S1, Supplementary Information). These observations indicate that the thermalized charge-carriers are extracted from the $\mathrm{MAPbI}_{3}$ films nearly at the similar rate. The reason for this behavior is discussed in further detail in the DFT section. 


\section{WILEY-VCH}

Peering into the extraction of hot carriers requires a finer probing time-scale (than hundreds of ps temporal resolution of TCSPC). Since the estimated hot-carrier diffusion length is on the order of $\sim 42 \mathrm{~nm}$ (see the detail calculation in Supplementary Note 3) in $\mathrm{MAPbI}_{3}$ films, the extraction of hot carrier is technically feasible. Hence, we studied the interfacial hot carrier extraction in the presence of ETL and HTL by fs-TA spectroscopy (exciting from the extraction layer sides ${ }^{[11]}$ ) and supported by DFT modelling. In order to make a fare comparison, we have also compared the fs-TA results for the both sample sides in Supporting Information (see Figure S5 and Supplementary Note 3).

Exploiting hot carriers in perovskite solar cells entails understanding their dynamics and extracting at the interface of $\mathrm{MAPbI}_{3}$ prior to their complete cooling. The hot carriers in pristine $\mathrm{MAPbI}_{3}$ show a non-equilibrium distribution when excited above the bandgap energy, as shown in Figure 3a. Under the same experimental conditions, the broadening of the high energy tails of the TA spectra slightly shrinks in the presence of the ETL (Figure 3b), and is completely diminished in the presence of the HTL (Figure 3c). This suggests that the hot carriers can be extracted from the $\mathrm{MAPbI}_{3}$ film using appropriate charge transport layers. However, as it can also be seen from Figure $3 \mathrm{~b}$ and $\mathrm{c}$, the efficiency of the hot carrier extraction is varies substantially depending on the layer's identity. By definition, hot carriers have excess kinetic energies at least more than $k_{B} T$ above the conduction and below the valence bands for electron and hole, respectively. Such hot carriers have higher carrier temperature, $T_{C}$, than the crystal lattice, which dissipates in a very short period of time (hundreds of fs) through a phonon emission process. The $T_{C}$ can be calculated by fitting the TA spectra (the fitting curves are provided in Supporting Information, see Figure S6) above the band edge (between $1.7 \mathrm{eV}$ and $2 \mathrm{eV}$ ) to a Boltzmann distribution ${ }^{[9 a, 9 b, 11-12]}$ :

$$
\Delta A[E] \propto e^{-\left(E-E_{f}\right) /\left(k_{B} T_{c}\right)}
$$




\section{WILEY-VCH}

Where, $E_{f}$ is the quasi-Fermi energy and $\mathrm{k}_{\mathrm{B}}$ is the Boltzmann constant. Figure $3 \mathrm{~d}$ shows the $T_{c}$ of $\mathrm{MAPbI}_{3}$ film drops from $\sim 1500 \mathrm{~K}$ to $600 \mathrm{~K}$ within $\sim 2$ ps after photoexcitation, implying that the excess energy of the carriers was released to the crystal lattice through non-radiative pathways. In the presence of $\mathrm{TiO}_{2}$, the initial temperature of the hot carrier also reduces with the progress of time following a similar pattern to the pristine $\mathrm{MAPbI}_{3}$. A markedly different behavior is seen when Spiro-OMeTAD is used as the extraction layer: we find that the initial temperature falls very rapidly and goes to $300 \mathrm{~K}$ within a $1 \mathrm{ps}$ timescale. In order to compare the hot carrier extraction across the three cases, we have plotted the normalized kinetics of all systems at $709 \mathrm{~nm}$ in Figure 4a. In the presence of Spiro-OMeTAD, the bleach recovers within less than 1 ps time scale, while for the perovskite alone and the perovskite with $\mathrm{TiO}_{2}$ the recovery within several picoseconds ps. This observation confirms that the excess energy of the hot hole efficiently was captured by the Spiro-OMeTAD before it thermalized to the crystal lattice of the $\mathrm{MAPbI}_{3}$. To see the effect of the pump photon energy on the hot carrier dynamics, the extraction of hot holes using different pump photon excitation is demonstrated (Figure S7). Upon 475-nm excitation, we do also see the hot carrier distribution as observed upon 370-nm excitation. In the presence of Spiro-OMeTAD, we found that the broadening of the high-energy tails was reduced, which indicates the extraction of the hot carrier by the Spiro-OMeTAD molecules. However, the efficiency of the hot hole extraction is not as high as was observed at 370-nm excitation. Also, to confirm the extraction of the holes, we performed further TA measurements in the NIR region (see Figure S8), which suggests slow relaxation of photoexcited carriers due to the capture of holes by Spiro-OMeTAD. However, it is difficult to conclude the nature of the hole from this measurement. Therefore, we conducted additional investigations by using theoretical modelling.

In order to shed light on the nature of hot carrier extractions, we performed DFT calculations on both MAI- and $\mathrm{PbI}_{2}$-terminated $\mathrm{MAPbI}_{3}$ (001) slab models (see Figure 4). For the extraction of hot carriers from the $\mathrm{MAPbI}_{3}$ film, we used the flat energy band alignment scheme (Figure 


\section{WILEY-VCH}

4b) although the interfacial band alignment will not be completely flat. ${ }^{[26]}$ Since, at high-energy excitation - as in the current study - the photogenerated hot carrier may overcome the barrier potential and can inject the electron into the conduction band of the $\mathrm{TiO}_{2}$ without facing any barrier. As shown in Figures $4 \mathrm{c}$ and $4 \mathrm{~d}$, for the $\mathrm{PbI}_{2}$-terminated slab, holes are preferentially delocalized on the top surface of $\mathrm{MAPbI}_{3}$ and electrons are delocalized along the whole $\mathrm{MAPbI}_{3}$ slab, which could overlap with the localized electronic clouds in the organic branches of SpiroOMeTAD or delocalized charges in $\mathrm{TiO}_{2}{ }^{\left[{ }^{[2]}\right.}$ In this case, the increased electrostatic potential at the interface of $\mathrm{MAPbI}_{3}$ and Spiro-OMeTAD can attract more holes than electrons. While in the case of MAI-terminated surface, the electrons reside well below the perovskite surface. Thus, the $\mathrm{PbI}_{2}$-terminated slab yields stronger electronic cloud overlap between the perovskite and charge transporting material, which results in the formation of a hybrid interface state upon hole/electron transfer. Noteworthy for both MAI- and $\mathrm{PbI}_{2}$-terminated surfaces, the deep-energy level (VBM-3.1 eV) shows electronic delocalization characteristic, providing a strong overlap with a localized charge of the highest occupied molecular orbital (HOMO) level from the SpiroOMeTAD molecule. For this reason, one can rationalize how holes could be easily extracted from the deep energy levels of $\mathrm{MAPbI}_{3}$ and transferred to the Spiro-OMeTAD. In sharp contrast, the electronic distribution for the high energy level $(\mathrm{VBM}+3.1 \mathrm{eV})$ is localized below the $\mathrm{MAPbI}_{3}$ surface, leading to the weak overlap with the charge of the conduction band the $\mathrm{TiO}_{2}$. This finding suggests that $\mathrm{MAPbI}_{3}$ can be used as efficient intermediates for extracting the hot holes after high energy excitation, but not for efficiently extracting hot electrons.

\section{Conclusion}

We systematically explored the ultrafast relaxation and hot charge-carrier extraction at the interface of $\mathrm{MAPbI}_{3}$ perovskite film. The time-resolved data demonstrate that the quasiequilibrium distribution of the hot carriers no longer exists in the presence of Spiro-OMeTAD, in contrast to the case of $\mathrm{MAPbI}_{3}$ alone and $\mathrm{MAPbI}_{3}$ with a $\mathrm{TiO}_{2}$ layer. This novel observation is supported by DFT calculations, which predict the existence of deep energy levels of 


\section{WILEY-VCH}

electronic delocalized characters that overlap with a localized charge valence band from the Spiro-OMeTAD molecule on the surface of $\mathrm{MAPbI}_{3}$. These findings elucidate the role of the deep-energy levels of $\mathrm{MAPbI}_{3}$ in enabling the hot hole extraction and transfer to the HTL; conversely, the absence of analogous energy states between the ETL and $\mathrm{MAPbI}_{3}$ is the main impediment for hot electron utilization. The striking difference in the behavior of hot carriers in the presence of the ETL versus the HTL reveals that the hot carrier extraction is closely related to the nature (electronic delocalization characteristics) of charge transport layers as well as the correspondingly aligned perovskite energy levels. This new finding illuminates a promising approach to design and fabricate perovskite solar cells that could utilize hot carrier.

\section{Experimental Section}

Chemicals: Lead iodide (99.999\%, ultradry) were purchased from AlfaAesar. DMF (anhydrous, 99.8\%), DMSO (anhydrous, $\geq 99 \%$ ) and GBL ( $\geq 99 \%$ ) were purchased from Sigma Aldrich. Methylammonium iodide (MAI) was purchased from Dyesol Limited (Australia). All salts and solvents were used as received without any further purification.

Materials and Methods: The glass substrates were cleaned by sonication in detergent, deionized water, acetone and isopropanol, sequentially for $10 \mathrm{~min}$ in each liquid, and then treated in UVOzone for $10 \mathrm{~min}$. To prepare compact $\mathrm{TiO}_{2}\left(\mathrm{c}-\mathrm{TiO}_{2}\right)$ solution, $369 \mu \mathrm{Ti}$-isopropoxide was added to $2.53 \mathrm{ml}$ of anhydrous ethanol and $35 \mu \mathrm{l}$ of $2 \mathrm{M} \mathrm{HCl}$ was added to $2.53 \mathrm{ml}$ of anhydrous isopropanol. The $\mathrm{HCl}$ solution was poured into Ti-isopropoxide solution drop wisely under vigorous stirring. The $\mathrm{c}-\mathrm{TiO}_{2}$ layer $(\sim 50 \mathrm{~nm})$ was deposited by spin coating with $3000 \mathrm{rpm}$ with ramp of $3000 \mathrm{rpm} \mathrm{s}^{-1}$ for $30 \mathrm{sec}$ on the pre-cleaned glass substrates. The $\mathrm{c}-\mathrm{TiO}_{2}$ coated substrate was sintered at $450{ }^{\circ} \mathrm{C}$ for $1 \mathrm{~h}$. The polycrystalline films of $\mathrm{MAPbI}_{3}$ perovskite (thickness was around $100 \mathrm{~nm}$ and determined by profilometer measurements) were prepared by the modified solvent engineering method. ${ }^{[28]}$ Namely, an equimolar ratio (1:1) of MAI and $\mathrm{PbI}_{2}$ was dissolved in a mixture of DMF-DMSO (9:1 by volume) to give $0.5 \mathrm{M}$ precursor 


\section{WILEY-VCH}

solution. After filtering through $0.45 \mu \mathrm{m}$ pore-size filter, the solution was spin coated on the glass or $\mathrm{c}-\mathrm{TiO}_{2}$ coated substrates at $6000 \mathrm{rpm}$ with ramp of $6000-\mathrm{rpm} \mathrm{s}^{-1}$ for $15 \mathrm{~s}$ under inert atmosphere. A $300 \mu 1$ of toluene was dropped at the $6^{\text {th }}$ second of spin coating. The films were annealed at $100{ }^{\circ} \mathrm{C}$ for $10 \mathrm{~min}$. The hole transport layer (HTL), Spiro-OMeTAD was deposited on the perovskite film via spin coating at $4000 \mathrm{rpm}$ for $45 \mathrm{~s}$ with a ramp of $4000 \mathrm{rpm} \mathrm{s}^{-1}$. The Spiro-OMeTAD solution was initially prepared by dissolving $72.5 \mathrm{mg}$ Spiro-OMeTAD, 28.5 $\mu \mathrm{L}$ 4-tert-butylpyridine and $17.5 \mu \mathrm{L}$ lithium (trifluoromathensulfonyl)imide solution (520 mg $\mathrm{ml}^{-1}$ in acetonitrile) in $1 \mathrm{~mL}$ chlorobenzene.

Steady-State Characterizations: The steady-state absorption were recorded using Edinburg F900 Spectrometer with an integrating sphere. Then Kubelka-Munk relation was used to convert the reflectance data to absorption spectra. The steady-state PL and the time-resolved PL were recorded using a FluoroMax ${ }^{\circledR}-4$ spectrofluorometer. Time-resolved PL measurement: The instrument works on the principle of time correlated single photon counting (TCSPC); a 450 $\mathrm{nm}$ laser pulses was used as the excitation light sources.

Transient absorption spectroscopy measurements: The pump-probe experiments were performed with femtosecond (fs) transient absorption spectroscopy with broadband capabilities and 120 fs resolution. Detailed information of the experimental setup can be found elsewhere. ${ }^{[29]}$ Briefly, an Ultrafast Systems Helios femtosecond transient absorption spectrometer equipped with UV-visible and near-infrared detectors was used to measure the perovskites samples in this study. All the samples were measured at room temperature and ambient conditions. The excitation pump pulses of 370 was generated after passing through a fraction of $800 \mathrm{~nm}$ beam into the spectrally tunable $(240-2600 \mathrm{~nm})$ optical parametric amplifier (Light conversion) using a few $\mu \mathrm{J}$ pulse energy of the fundamental output of a Ti:sapphire fs regenerative amplifier operating at $800 \mathrm{~nm}$ with $35 \mathrm{fs}$ pulses and a repetition rate of $1 \mathrm{kHz}$. The probe pulses (UV visible and NIR wavelength continuum, white light) were generated by 


\section{WILEY-VCH}

passing another fraction of the $800 \mathrm{~nm}$ pulses through the 2-mm thick sapphire crystal. Before white light generation, the $800 \mathrm{~nm}$ amplified pulses were passed through a motorized delay stage. Depending on the movement of delay stage, the transient species were detected at different time scales following excitation. The white light was split into two beams (named as signal and reference) and focused on two fiber optics for the improvement of the signal to noise ratio. The excitation pump pulses were spatially overlapped with the probe pulses on the samples after passing through a synchronized mechanical chopper $(500 \mathrm{~Hz})$, which blocked an alternative pump pulses. The fluence of the pump power was adjusted by using neutral density (ND) filters. All the fs-TA experiments were performed under identical ambient conditions at room temperature.

Computational Methods: Density functional theory (DFT) calculations were performed with a generalized gradient approximation (GGA)/Perdew-Burke-Ernzerhof (PBE) level using the projector-augmented wave (PAW) method, as implemented in the VASP code. ${ }^{[30]}$ For the tetragonal-phase bulk $\mathrm{MAPbI}_{3}$, the electronic plane-wave cutoff energy was set to $500 \mathrm{eV}$ and the van der Waals functional (vdW-DF) was used during the crystal structural optimizations. Monkhorst-Pack-type K-meshes of $6 \times 6 \times 6$ for the bulk $\mathrm{MAPbI}_{3}$ and $6 \times 6 \times 1$ for slabs exposing the (001) surface with both MAI- and $\mathrm{PbI}_{2}$ - terminations were used. All the symmetric slabs were separated by the top vacuum layer $(\sim 15 \AA)$ to prevent spurious inter-slab interactions. All the geometries were optimized until all forces on all atoms were smaller than $0.01 \mathrm{eV} / \AA$. The Heyd-Scuseria-Ernzerhof hybrid functional (HSE06) including spin-orbit coupling (SOC) was used to calculate the density of states and corresponding charge densities. All of the three-dimensional representations of crystal structures and charge densities were obtained using VESTA.

\section{Supporting Information}

Supporting Information is available from the Wiley Online Library or from the author. 


\section{WILEY-VCH}

\section{Acknowledgements}

The authors acknowledge the financial support of King Abdullah University of Science and Technology (KAUST). I. Dursun and P. Maity contributed equally to this work.

Received: ((will be filled in by the editorial staff))

Revised: ((will be filled in by the editorial staff))

Published online: ((will be filled in by the editorial staff))

\section{References}

[1] a) M. A. Green, Prog. Photovoltaics 2001, 9, 123; b) Y. Liyan, T. B. Alexander, G. L. David, W. Tao, Rep. Prog. Phys 2016, 79, 026501.

[2] a) M. Graetzel, R. A. J. Janssen, D. B. Mitzi, E. H. Sargent, Nature 2012, 488, 304; b) W. Wang, M. T. Winkler, O. Gunawan, T. Gokmen, T. K. Todorov, Y. Zhu, D. B. Mitzi, Adv. Energy Mater. 2014, 4, 1301465; c) C. J. Brabec, N. S. Sariciftci, J. C. Hummelen, Adv. Funct. Mater. 2001, 11, 15; d) D. B. Mitzi, O. Gunawan, T. K. Todorov, K. Wang, S. Guha, Sol. Energy Mater Sol. Cells 2011, 95, 1421.

[3] a) W. A. Tisdale, K. J. Williams, B. A. Timp, D. J. Norris, E. S. Aydil, X.-Y. Zhu, Science 2010, 328, 1543; b) R. T. Ross, A. J. Nozik, J. Appl. Phys. 1982, 53, 3813; c) H.-H. Fang, S. Adjokatse, S. Shao, J. Even, M. A. Loi, Nat. Commun. 2018, 9, 243.

[4] a) G. Xing, N. Mathews, S. Sun, S. S. Lim, Y. M. Lam, M. Grätzel, S. Mhaisalkar, T. C. Sum, Science 2013, 342, 344; b) S. D. Stranks, G. E. Eperon, G. Grancini, C. Menelaou, M. J. P. Alcocer, T. Leijtens, L. M. Herz, A. Petrozza, H. J. Snaith, Science 2013, 342, 341; c) D. Shi, V. Adinolfi, R. Comin, M. Yuan, E. Alarousu, A. Buin, Y. Chen, S. Hoogland, A. Rothenberger, K. Katsiev, Y. Losovyj, X. Zhang, P. A. Dowben, O. F. Mohammed, E. H. Sargent, O. M. Bakr, Science 2015, 347, 519; d) J. S. Manser, M. I. Saidaminov, J. A. Christians, O. M. Bakr, P. V. Kamat, Acc. Chem. Res. 2016, 49, 330; e) Y. Yang, M. Yang, David T. Moore, Y. Yan, Elisa M. Miller, K. Zhu, Matthew C. Beard, Nat. Energy 2017, 2, 16207; f) B. Saparov, D. B. Mitzi, Chem. Rev. 2016, 116, 4558; g) D. W. de Quilettes, S. M. Vorpahl, S. D. Stranks, H. Nagaoka, G. E. Eperon, M. E. Ziffer, H. J. Snaith, D. S. Ginger, Science 2015, 348, 683; h) National Renewable Energy Laboratory (NREL), Best Research-Cell Efficiencies, http://www.nrel.gov//ncpv/images/efficiency-chart.jpg, accessed 2018; i) N. J. Jeon, H. Na, E. H. Jung, T.-Y. Yang, Y. G. Lee, G. Kim, H.-W. Shin, S. Il Seok, J. Lee, J. Seo, Nat. Energy 2018, 3, 682.

[5] M. I. Saidaminov, V. Adinolfi, R. Comin, A. L. Abdelhady, W. Peng, I. Dursun, M. Yuan, S. Hoogland, E. H. Sargent, O. M. Bakr, Nat. Commun. 2015, 6, 8724.

[6] Q. Chen, J. Wu, X. Ou, B. Huang, J. Almutlaq, A. A. Zhumekenov, X. Guan, S. Han, L. Liang, Z. Yi, J. Li, X. Xie, Y. Wang, Y. Li, D. Fan, D. B. L. Teh, A. H. All, O. F. Mohammed, O. M. Bakr, T. Wu, M. Bettinelli, H. Yang, W. Huang, X. Liu, Nature 2018, 561, 88 .

[7] a) Z.-K. Tan, R. S. Moghaddam, M. L. Lai, P. Docampo, R. Higler, F. Deschler, M. Price, A. Sadhanala, L. M. Pazos, D. Credgington, F. Hanusch, T. Bein, H. J. Snaith, R. H. Friend, Nat. Nanotechnol. 2014, 9, 687; b) Z. Xiao, R. A. Kerner, L. Zhao, N. L. Tran, K. M. Lee, T.-W. Koh, G. D. Scholes, B. P. Rand, Nat. Photonics 2017, 11, 108; c) H. Kim, L. Zhao, J. S. Price, A. J. Grede, K. Roh, A. N. Brigeman, M. Lopez, B. P. Rand, N. C. Giebink, Nat. Commun. 2018, 9, 4893.

[8] a) S. Yakunin, L. Protesescu, F. Krieg, M. I. Bodnarchuk, G. Nedelcu, M. Humer, G. De Luca, M. Fiebig, W. Heiss, M. V. Kovalenko, Nat. Commun. 2015, 6, 8056; b) F. Deschler, M. Price, S. Pathak, L. E. Klintberg, D.-D. Jarausch, R. Higler, S. Hüttner, T. Leijtens, S. D. Stranks, H. J. Snaith, M. Atatüre, R. T. Phillips, R. H. Friend, J. 


\section{WILEY-VCH}

Phys. Chem. Lett. 2014, 5, 1421; c) Y. Jia, R. A. Kerner, A. J. Grede, B. P. Rand, N. C. Giebink, Nat. Photonics 2017, 11, 784; d) H. Zhu, Y. Fu, F. Meng, X. Wu, Z. Gong, Q. Ding, M. V. Gustafsson, M. T. Trinh, S. Jin, X. Y. Zhu, Nat. Mater. 2015, 14, 636; e) S. W. Eaton, M. Lai, N. A. Gibson, A. B. Wong, L. Dou, J. Ma, L.-W. Wang, S. R. Leone, P. Yang, Proc. Natl. Acad. Sci. U.S.A. 2016, 113, 1993; f) J. Pan, S. P. Sarmah, B. Murali, I. Dursun, W. Peng, M. R. Parida, J. Liu, L. Sinatra, N. Alyami, C. Zhao, E. Alarousu, T. K. Ng, B. S. Ooi, O. M. Bakr, O. F. Mohammed, J. Phys. Chem. Lett. 2015, 6, 5027.

[9] a) M. B. Price, J. Butkus, T. C. Jellicoe, A. Sadhanala, A. Briane, J. E. Halpert, K. Broch, J. M. Hodgkiss, R. H. Friend, F. Deschler, Nat. Commun. 2015, 6, 8420; b) Y. Yang, D. P. Ostrowski, R. M. France, K. Zhu, J. van de Lagemaat, J. M. Luther, M. C. Beard, Nat. Photonics 2015, 10, 53; c) Z. Guo, Y. Wan, M. Yang, J. Snaider, K. Zhu, L. Huang, Science 2017, 356, 59; d) J. Fu, Q. Xu, G. Han, B. Wu, C. H. A. Huan, M. L. Leek, T. C. Sum, Nat. Commun. 2017, 8, 1300; e) G. J. Hedley, C. Quarti, J. Harwell, O. V. Prezhdo, D. Beljonne, I. D. W. Samuel, Sci. Rep. 2018, 8, 8115; f) S. Nah, B. M. Spokoyny, C. M. M. Soe, C. C. Stoumpos, M. G. Kanatzidis, E. Harel, Nano Lett. 2018, 18, 1044; g) B. Anand, S. Sampat, E. O. Danilov, W. Peng, S. M. Rupich, Y. J. Chabal, Y. N. Gartstein, A. V. Malko, Phys. Rev. B 2016, 93, 161205.

[10] M. Li, J. Fu, Q. Xu, T. C. Sum, Adv. Mater. 0, 1802486.

[11] M. Li, S. Bhaumik, T. W. Goh, M. S. Kumar, N. Yantara, M. Grätzel, S. Mhaisalkar, N. Mathews, T. C. Sum, Nat. Commun. 2017, 8, 14350.

[12] Q. Shen, T. S. Ripolles, J. Even, Y. Zhang, C. Ding, F. Liu, T. Izuishi, N. Nakazawa, T. Toyoda, Y. Ogomi, S. Hayase, J. Energy Chem. 2018, 27, 1170.

[13] S. Sarkar, V. K. Ravi, S. Banerjee, G. R. Yettapu, G. B. Markad, A. Nag, P. Mandal, Nano Lett. 2017, 17, 5402.

[14] Y. Kanemitsu, J. Mater. Chem. C 2017, 5, 3427.

[15] J. S. Manser, P. V. Kamat, Nat. Photonics 2014, 8, 737.

[16] M. T. Trinh, X. Wu, D. Niesner, X. Y. Zhu, J. Mater. Chem. A 2015, 3, 9285.

[17] J. Yang, X. Wen, H. Xia, R. Sheng, Q. Ma, J. Kim, P. Tapping, T. Harada, T. W. Kee, F. Huang, Y.-B. Cheng, M. Green, A. Ho-Baillie, S. Huang, S. Shrestha, R. Patterson, G. Conibeer, Nat. Commun. 2017, 8, 14120.

[18] a) J. M. Richter, F. Branchi, F. Valduga de Almeida Camargo, B. Zhao, R. H. Friend, G. Cerullo, F. Deschler, Nat. Commun. 2017, 8, 376; b) T. Ghosh, S. Aharon, L. Etgar, S. Ruhman, J. Am. Chem. Soc 2017, 139, 18262.

[19] Y. Rosenwaks, M. C. Hanna, D. H. Levi, D. M. Szmyd, R. K. Ahrenkiel, A. J. Nozik, Phys. Rev. B 1993, 48, 14675.

[20] C. Wehrenfennig, M. Liu, H. J. Snaith, M. B. Johnston, L. M. Herz, APL Materials 2014, 2.

[21] a) L. Q. Phuong, Y. Yamada, M. Nagai, N. Maruyama, A. Wakamiya, Y. Kanemitsu, J. Phys. Chem. Lett. 2016, 7, 2316; b) W. Wang, Y. Li, X. Wang, Y. Liu, Y. Lv, S. Wang, K. Wang, Y. Shi, L. Xiao, Z. Chen, Q. Gong, Sci. Rep. 2017, 7, 14760; c) S. Ghosh, Q. Shi, B. Pradhan, P. Kumar, Z. Wang, S. Acharya, S. K. Pal, T. Pullerits, K. J. Karki, J. Phys. Chem. Lett. 2018, 9, 4245; d) Y. Yamada, T. Nakamura, M. Endo, A. Wakamiya, Y. Kanemitsu, J. Am. Chem. Soc 2014, 136, 11610; e) S. D. Stranks, V. M. Burlakov, T. Leijtens, J. M. Ball, A. Goriely, H. J. Snaith, Phys. Rev. Appl. 2014, 2, 034007; f) T. Hakamata, K. Shimamura, F. Shimojo, R. K. Kalia, A. Nakano, P. Vashishta, Sci. Rep. 2016, 6, 19599; g) Y. Li, W. Wang, X. Wang, S. Wang, Y. Shi, Q. Gong, J. Mater. Chem. C 2017, 5, 3283; h) Y. Yamada, T. Yamada, A. Shimazaki, A. Wakamiya, Y. Kanemitsu, J. Phys. Chem. Lett. 2016, 7, 1972.

[22] A. D. Wright, C. Verdi, R. L. Milot, G. E. Eperon, M. A. Pérez-Osorio, H. J. Snaith, F. Giustino, M. B. Johnston, L. M. Herz, Nat. Commun. 2016, 7, 11755. 


\section{WILEY-VCH}

[23] P.-A. Mante, C. C. Stoumpos, M. G. Kanatzidis, A. Yartsev, Nat. Commun. 2017, 8, 14398.

[24] Y. Yamada, T. Yamada, L. Q. Phuong, N. Maruyama, H. Nishimura, A. Wakamiya, Y. Murata, Y. Kanemitsu, J. Am. Chem. Soc 2015, 137, 10456.

[25] V. D'Innocenzo, A. R. Srimath Kandada, M. De Bastiani, M. Gandini, A. Petrozza, J. Am. Chem. Soc 2014, 136, 17730.

[26] G. Xing, B. Wu, S. Chen, J. Chua, N. Yantara, S. Mhaisalkar, N. Mathews, T. C. Sum, Small 2015, 11, 3606.

[27] a) E. Mosconi, E. Ronca, F. De Angelis, J. Phys. Chem. Lett. 2014, 5, 2619; b) J. Yin, D. Cortecchia, A. Krishna, S. Chen, N. Mathews, A. C. Grimsdale, C. Soci, J. Phys. Chem. Lett. 2015, 6, 1396.

[28] N. J. Jeon, J. H. Noh, Y. C. Kim, W. S. Yang, S. Ryu, S. I. Seok, Nat. Mater. 2014, $13,897$.

[29] a) R. Begum, M. R. Parida, A. L. Abdelhady, B. Murali, N. M. Alyami, G. H. Ahmed, M. N. Hedhili, O. M. Bakr, O. F. Mohammed, J. Am. Chem. Soc 2017, 139, 731; b) J. Sun, W. Yu, A. Usman, T. T. Isimjan, S. Dgobbo, E. Alarousu, K. Takanabe, O. F. Mohammed, J. Phys. Chem. Lett. 2014, 5, 659.

[30] a) G. Kresse, D. Joubert, Phys. Rev. B 1999, 59, 1758; b) G. Kresse, J. Furthmüller, Comput. Mater. Sci. 1996, 6, 15. 


\section{WILEY-VCH}
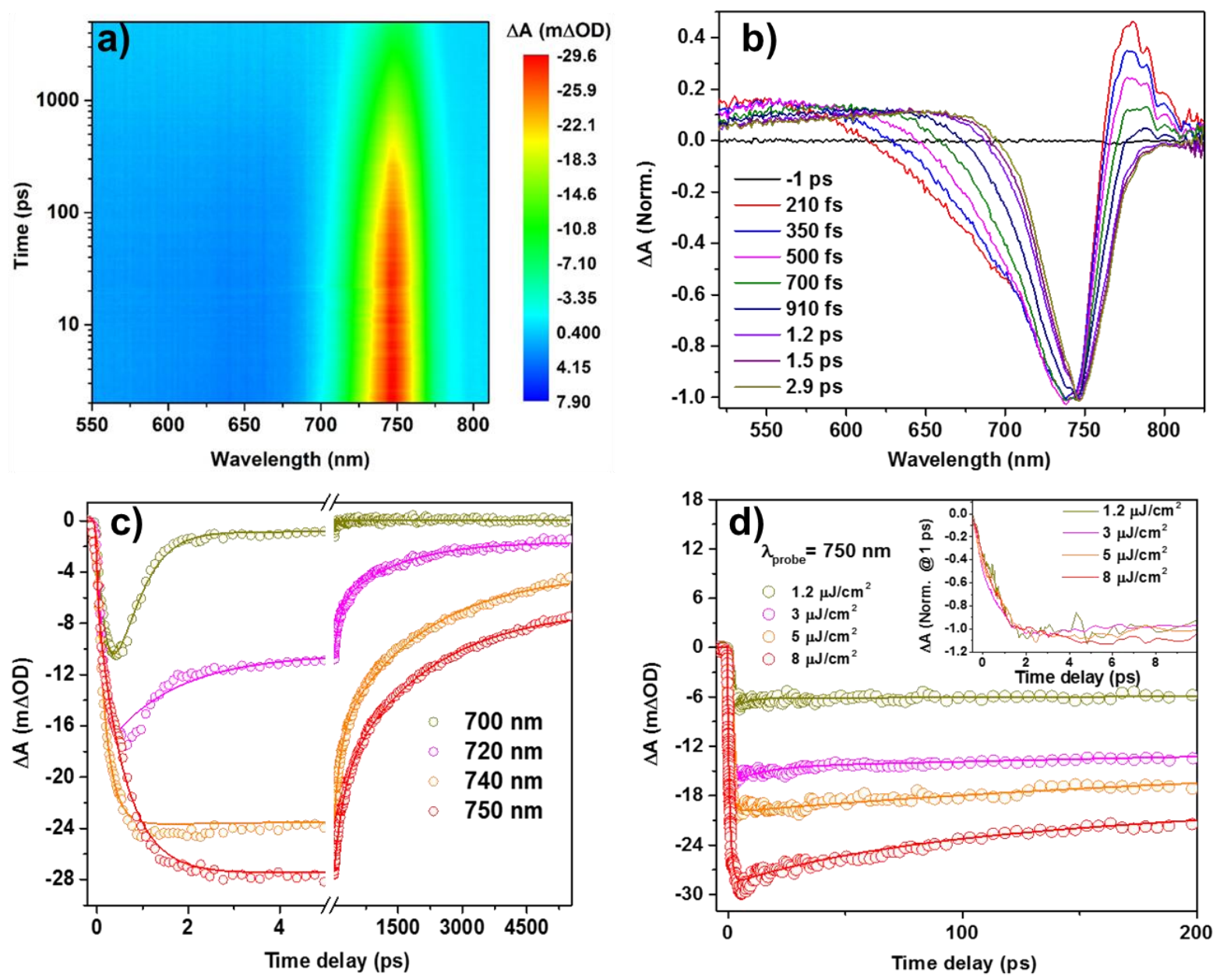

Figure 1. fs-TA spectra of $\mathrm{MAPbI}_{3}$ perovskite. a) 2D color plot of TA spectrum, b) normalized spectra at short time delay and, c) bleach recovery kinetics at different wavelengths of $\mathrm{MAPbI}_{3}$ perovskite in response to $370 \mathrm{~nm}$ optical pulses with $3 \mu \mathrm{J} / \mathrm{cm}^{2}$ pump fluence. d) Recovery of the bleaching signal at $750 \mathrm{~nm}$ as a function of pump fluence (carrier densities vary from 2.16 $\mathrm{x} 10^{17} \mathrm{~cm}^{-3}$ to $1.44 \times 10^{18} \mathrm{~cm}^{-3}$ ). The inset shows the normalized bleach kinetics at short timescale. 


\section{WILEY-VCH}

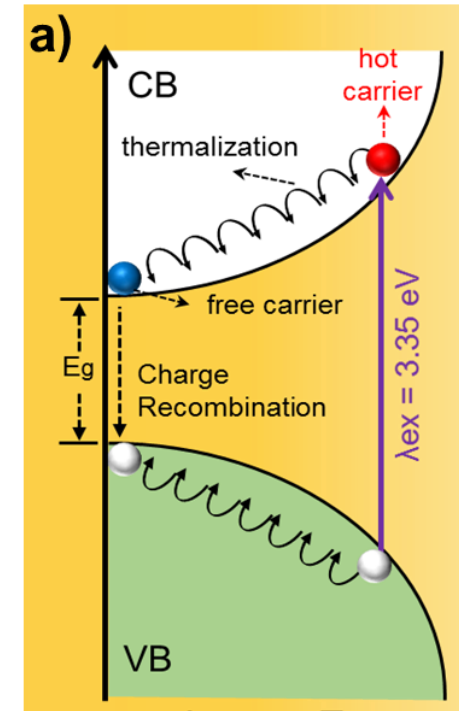

$h v_{1} \gg E_{g}$

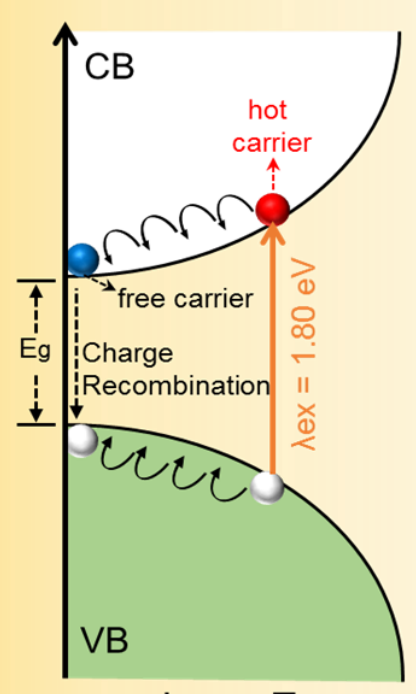

$\mathrm{hv}_{2}>\mathrm{Eg}$

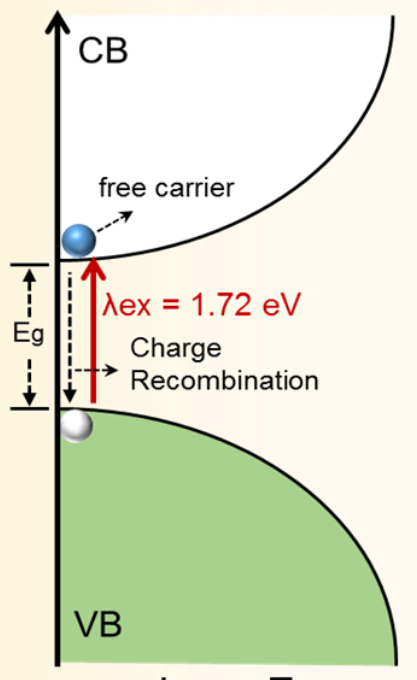

$\mathrm{hv}_{3} \sim \mathrm{Eg}_{\mathrm{g}}$
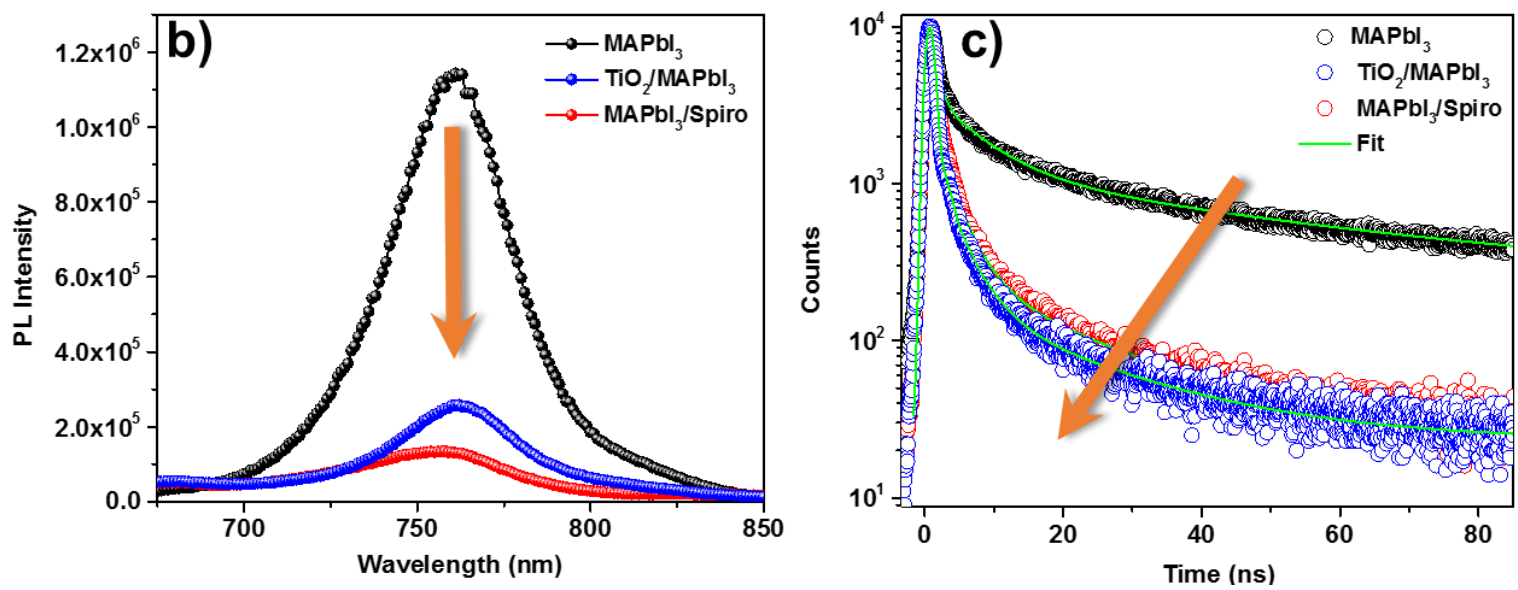

Figure 2. a) Schematic diagram of electron relaxation processes in $\mathrm{MAPbI}_{3}$ with three different pump excitation values: with excess energy, at close to band edge and band edge $(370,690$, and $720 \mathrm{~nm}$ optical excitations). b) Steady-state $\left(\lambda_{\mathrm{ex}}=450 \mathrm{~nm}\right)$ and c) time-resolved PL spectra of the pristine $\mathrm{MAPbI}_{3}$ (black), $\mathrm{MAPbI}_{3}-\mathrm{TiO}_{2}$ (blue), Spiro-OMeTAD -MAPbI 3 (red). The timeresolved PL decay was monitored at $760 \mathrm{~nm}$ after $450 \mathrm{~nm}$ laser excitation. 


\section{WILEY-VCH}
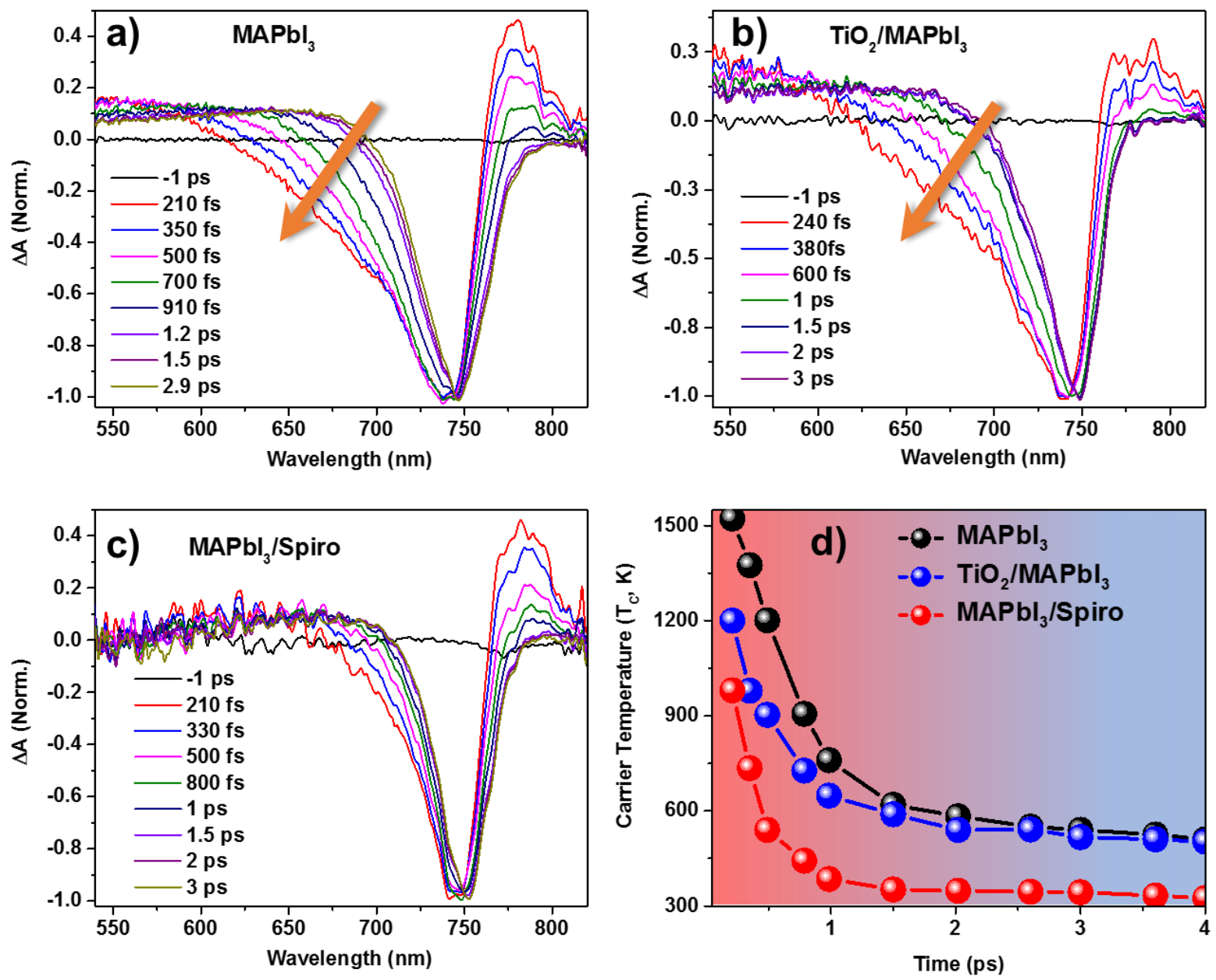

Figure 3. Normalized fs-TA spectra of $\mathrm{MAPbI}_{3}$ film (a), $\mathrm{MAPbI}_{3}-\mathrm{TiO}_{2}$ (b), and $\mathrm{MAPbI}_{3}-\mathrm{Spiro}$ (c) in response to a 370-nm excitation with a fixed pump fluence of $3 \mu \mathrm{J} / \mathrm{cm}^{2}$. (d) Hot carrier cooling as a function of delay time, extracted from (a), (b), and (c) (films are excited from the extraction layer side). 


\section{WILEY-VCH}
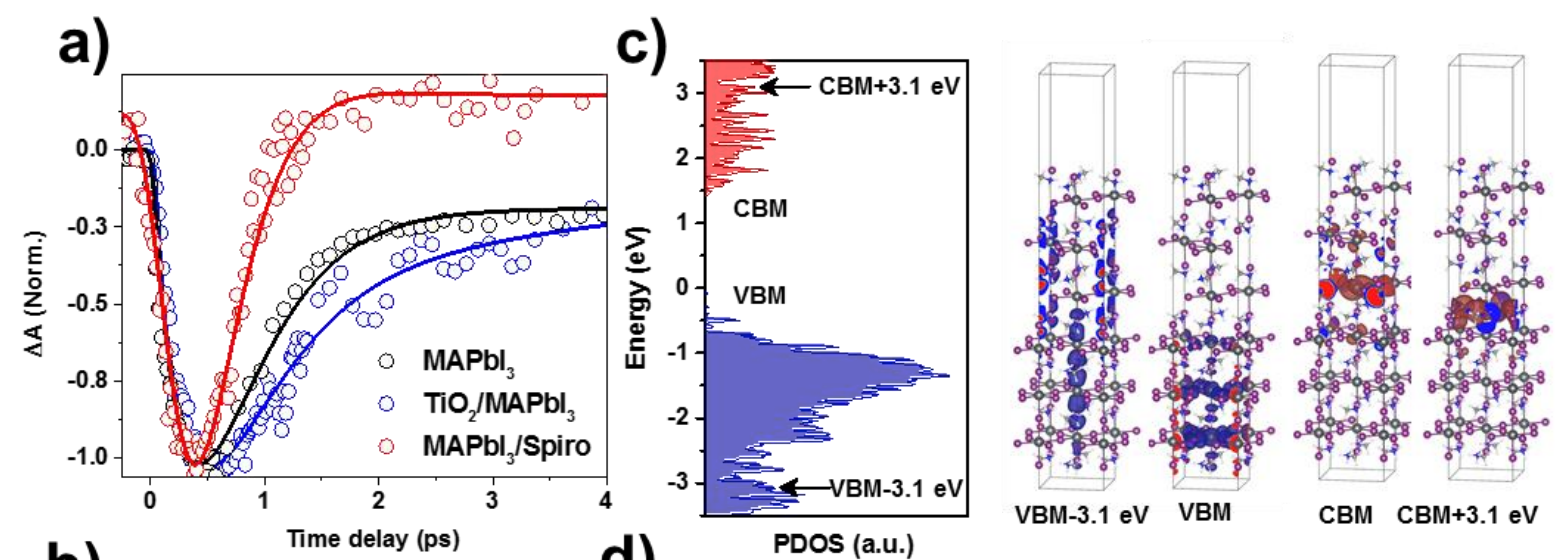

b)

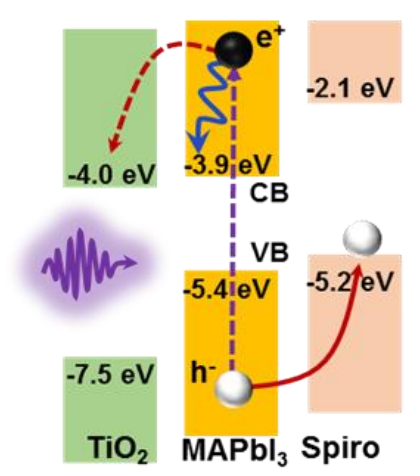

d)
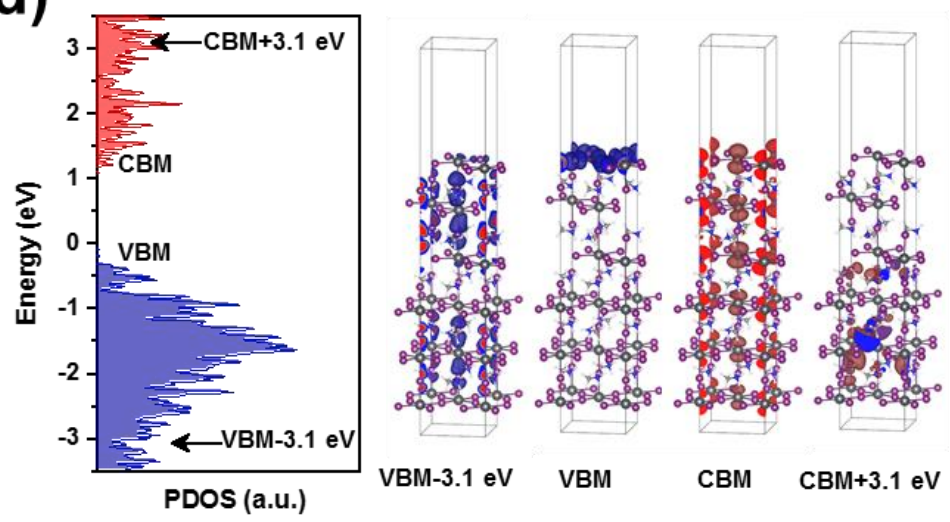

Figure 4. a) Normalized fs-TA kinetics at $709 \mathrm{~nm}$ of the pristine $\mathrm{MAPbI}_{3}$ film, $\mathrm{TiO}_{2} / \mathrm{MAPbI}_{3}$, and $\mathrm{MAPbI}_{3} / \mathrm{Sp}$ iro-OMeTAD in response to a 370-nm excitation with a fixed pump fluence of $3 \mu \mathrm{J} / \mathrm{cm}^{2}$. b) Schematic of energy alignment and competition of thermalization and hot charge injection processes between interfaces of $\mathrm{TiO}_{2} / \mathrm{MAPbI}_{3}$ and $\mathrm{MAPbI}_{3} / \mathrm{Spiro}-\mathrm{OMeTAD}$; projected density of states and three-dimensional electronic charge distributions (an isovalue of $5 \times 10^{-4} \mathrm{e} / \AA^{3}$ ) of VBM-3.1 eV, VBM, CBM and VBM+3.1 eV for c) MAI-terminated and d) $\mathrm{PbI}_{2}$-terminated $\mathrm{MAPbI}_{3}$ (001) slab calculated at $\mathrm{HSE}+\mathrm{SOC}$ level of theory (the VBM is set at zero energy). 


\section{WILEY-VCH}

Interfacial hot carrier extraction in MAPbI 3 perovskite films is explored by femtosecond transient absorption spectroscopy. Compared to hot electrons, the extraction of hot holes is more efficient at the interface of $\mathrm{MAPbI}_{3}$.

Keywords: Halide perovskite, hot carriers, carrier temperature, carrier extraction, density functional theory

Ibrahim Dursun, $\uparrow$ Partha Maity, $\dagger$ Jun Yin, Bekir Turedi, Ayan A. Zhumekenov, Kwang Jae Lee, Omar F. Mohammed,* and Osman M. Bakr,*

Why are Hot Holes Easier to Extract than Hot Electrons from Methylammonium Lead Iodide Perovskite?

TOC figure

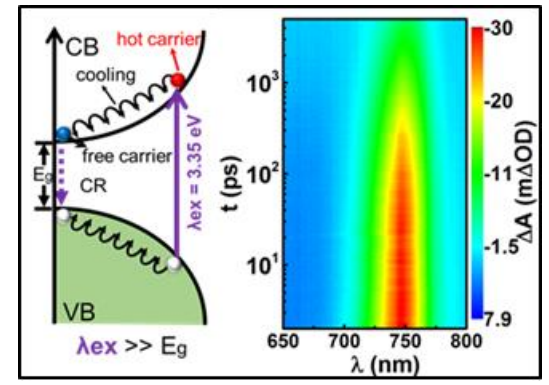




\section{WILEY-VCH}

Copyright WILEY-VCH Verlag GmbH \& Co. KGaA, 69469 Weinheim, Germany, 2018.

\section{Supporting Information}

Why are Hot Holes Easier to Extract than Hot Electrons from Methylammonium Lead Iodide Perovskite?

Ibrahim Dursun, $\uparrow$ Partha Maity, $\dagger$ Jun Yin, Bekir Turedi, Ayan A. Zhumekenov, Kwang Jae Lee, Omar F. Mohammed, * and Osman M. Bakr, *

Supplementary Note 1: Femtosecond pump probe spectroscopy details

In this study, we varied the pump fluence from $1.2 \mu \mathrm{J} / \mathrm{cm}^{2}$ to $8 \mu \mathrm{J} / \mathrm{cm}^{2}$. Considering the excited beam spot size (diameter) $\sim 0.04 \mathrm{~cm}$, we calculated the fluence of the pump pulse. One example below,

Measured power $=3.9 \mu \mathrm{W}$

Per pulse Energy $(\mathrm{E})=3.9 \mathrm{~nJ}($ pulse repetition rate $=1 \mathrm{KHz})$

Beam sport size $=\sim 0.04 \mathrm{~cm}($ Diameter $)$

Beam sport radius $(\mathrm{r})=0.02 \mathrm{~cm}$

Area of the beam spot (A) $=\pi r^{2}$

$$
=1.26 \times 10^{-3} \mathrm{~cm}^{2}
$$

Fluence $=\frac{E}{A}=\frac{3.9}{1.26 \times 10^{-3}} \mathrm{~nJ} / \mathrm{cm}^{2}$

$$
=3.09 \mu \mathrm{J} / \mathrm{cm}^{2}
$$


Supplementary Note 2: Carrier density calculation

Pump fluence $(\mathrm{j})=1 \mu \mathrm{J} / \mathrm{cm}^{2}$

$\mathrm{E}=\operatorname{nh}(\mathrm{C} / \lambda), \lambda=370 \mathrm{~nm}$, Number of photons (n)

$\mathrm{n}=1.8 \times 10^{12}$ photon $/ \mathrm{cm}^{2}$

Carrier density $\left(\mathrm{n}_{0}\right)=\mathrm{j} \times \alpha$

Where, $\mathrm{j}=$ pump fluence

$$
\alpha=\text { absorption coefficient at excitation wavelength }
$$

The measured pump fluence varies $1.2-8 \mu \mathrm{J} / \mathrm{cm}^{2}$ (which equivalent to $1.44 \times 10^{13}$ photon $/ \mathrm{cm}^{2}$, considering $8 \mu \mathrm{J} / \mathrm{cm}^{2}$ )

Now, carrier density $\left(\mathrm{n}_{0}\right)=1.44 \times 10^{13} \times 1 \times 10^{5} \mathrm{~cm}^{-3}$

$$
=1.44 \times 10^{18} \mathrm{~cm}^{-3} \text { (Carrier density correspond to the pump fluence } 8
$$

$\left.\mu \mathrm{J} / \mathrm{cm}^{2}\right)$

(Considering the absorption coefficient of $\mathrm{MAPbI}_{3}$ film ${ }^{1} 3 \mathrm{eV} \sim 1 \times 10^{5} \mathrm{~cm}^{-1}$ )

Supplementary Note 3: Calculation of hot carrier diffusion length

Hot carrier diffusion length, $L_{\text {hot }}=\sqrt{D_{\text {hot }} \times \tau_{\text {hot }}}$

Where, $D_{\text {hot }}$ and $\tau_{\text {hot }}$ are diffusion coefficient and lifetime of the hot carrier, respectively.

$D_{\text {hot }}$ can be written as

$$
D_{h o t}=\frac{\mu \times k_{B} \times T_{c}}{e}
$$

Where,

$\mu=$ mobility of the hot carrier $\left(500 \mathrm{~cm}^{2} \mathrm{~V}^{-1} \mathrm{~s}^{-1}\right)^{2}$

$\mathrm{k}_{\mathrm{B}}=$ Boltzmann constant $\left(1.38 \times 10^{-23} \mathrm{~J} \mathrm{~K}^{-1}\right)$

$\mathrm{T}_{\mathrm{c}}=$ hot carrier temperature $(750 \mathrm{~K})$

and $\mathrm{e}=$ electronic charge $\left(1.602 \times 10^{-19} \mathrm{~J} \mathrm{~V}^{-1}\right)$

Therefore, $D_{\text {hot }}=32.3 \mathrm{~cm}^{2} \mathrm{~s}^{-1}$ 


\section{WILEY-VCH}

Considering the cooling temperature of the hot carrier $540 \mathrm{fs}$,

$L_{h o t}=\sqrt{32.3 \times 540 \times 10^{-15}} \mathrm{~cm}$

$=4.2 \times 10^{-6} \mathrm{~cm}$

$=42 \mathrm{~nm}$

Effect of excitation direction: Both sides TA results are almost similar for $\mathrm{TiO}_{2} / \mathrm{MAPbI}_{3}$ films; however, hot carrier extraction is more efficient when we excited from extraction layer side of the $\mathrm{MAPbI}_{3} / \mathrm{Spiro}$ due to the less diffusion of laser pulse excitation.

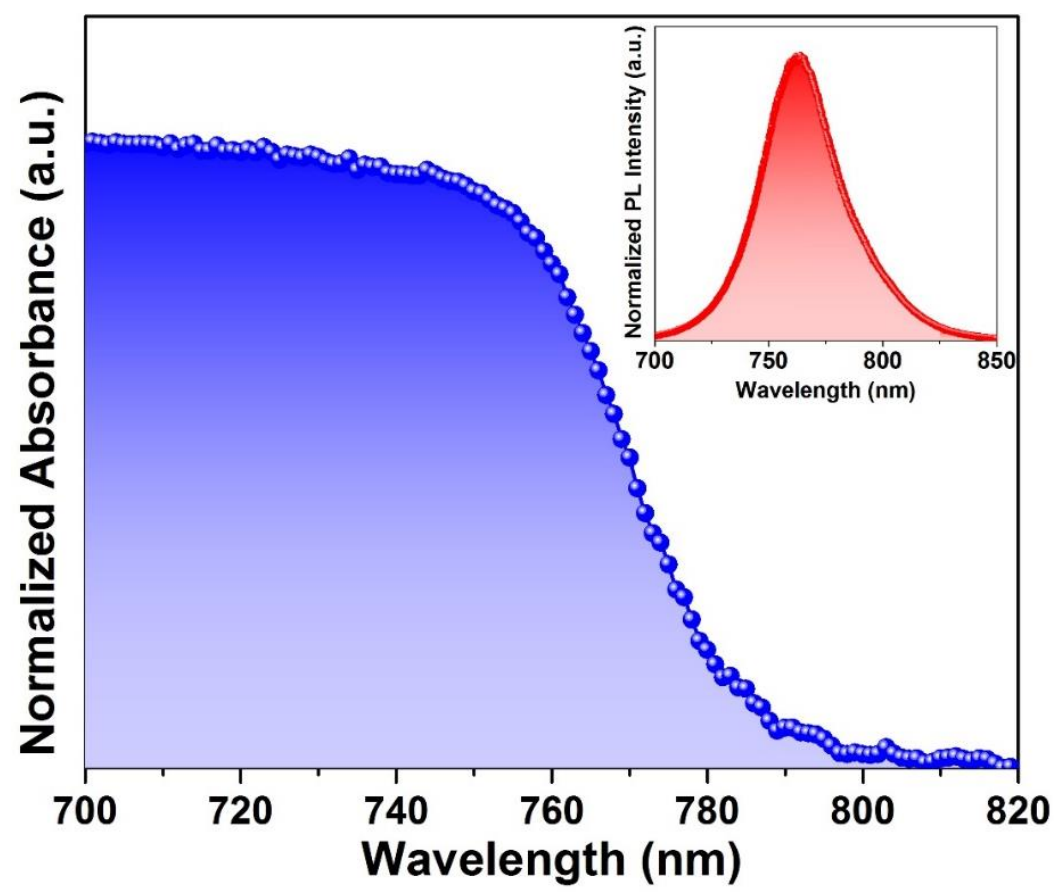

Figure S1: Steady state normalized absorption and photoluminescence spectra of $\mathrm{MAPbI}_{3}$. 


\section{WILEY-VCH}

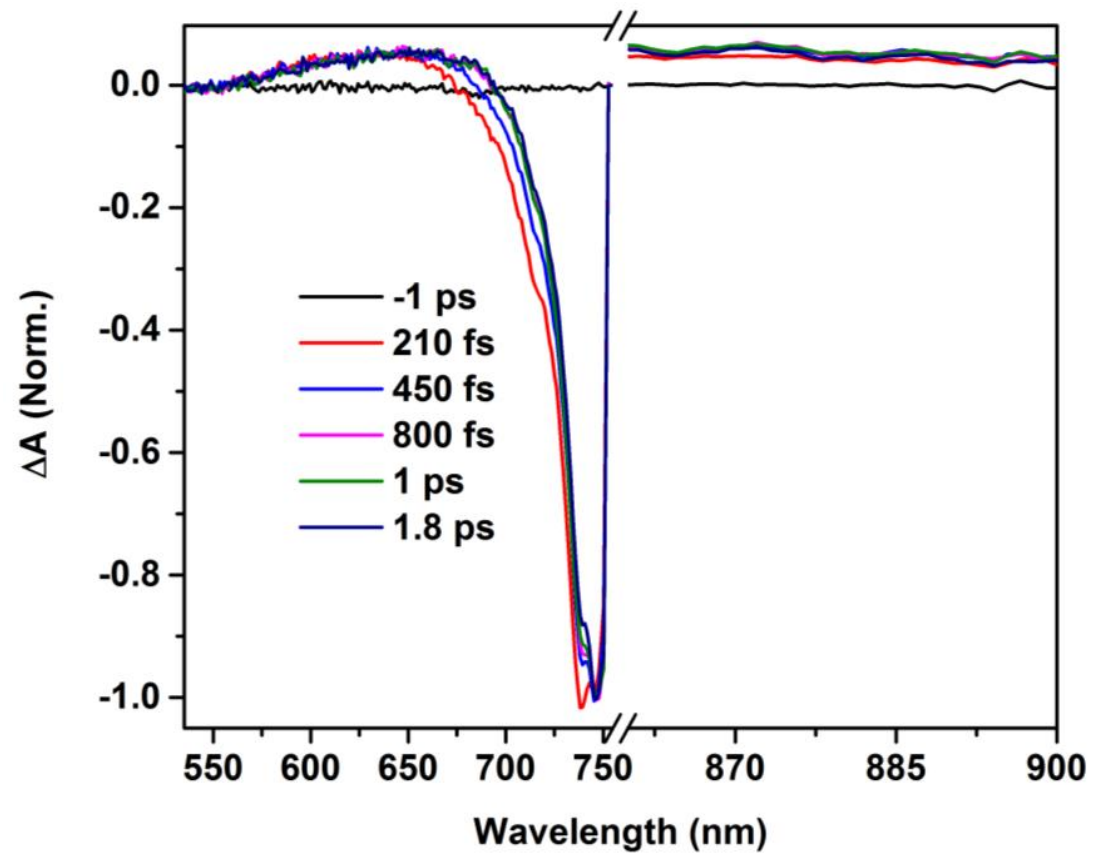

Figure S2: Normalized fs-TA spectra of $\mathrm{MAPbI}_{3}$ film in response to a $720-\mathrm{nm}$ excitation with a fixed pump fluence of $3 \mu \mathrm{J} / \mathrm{cm}^{2}$.

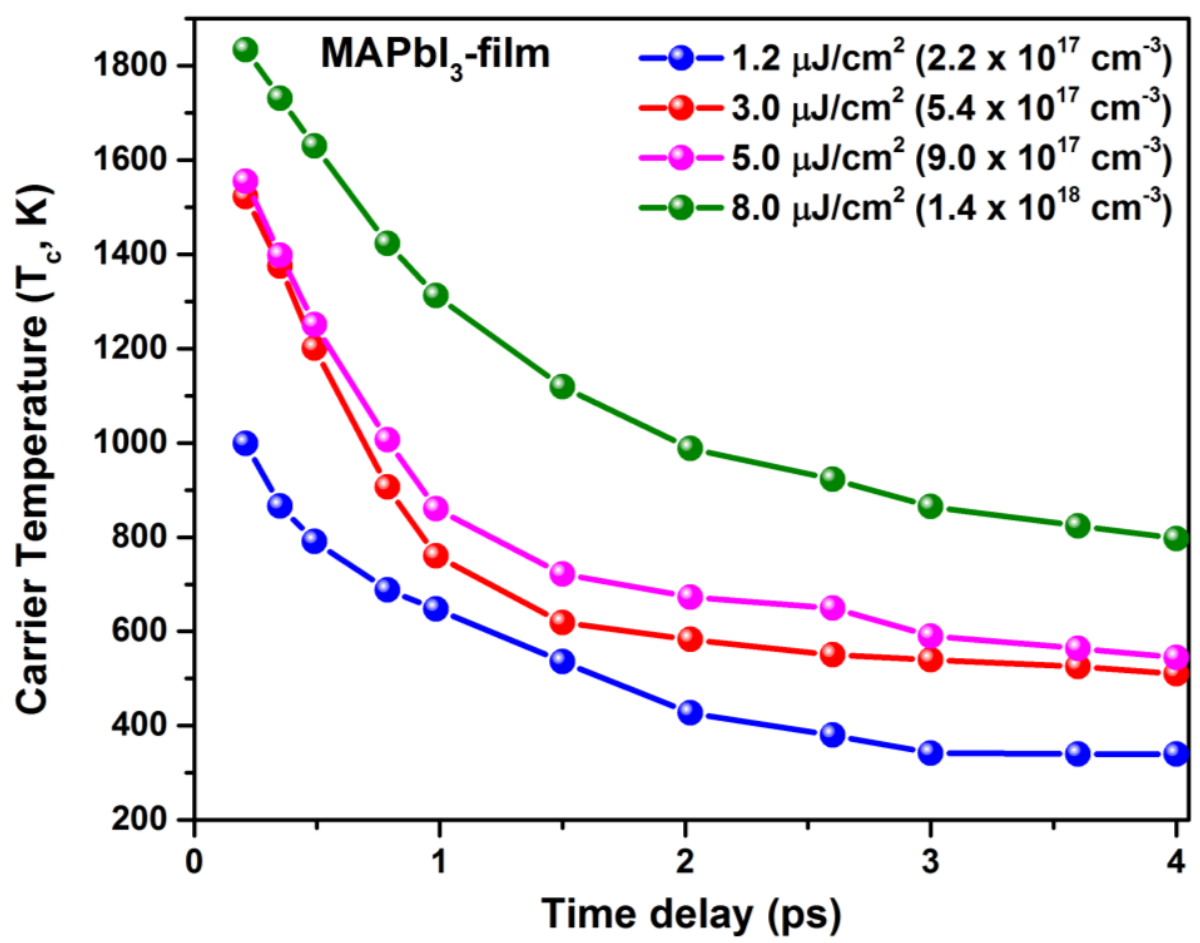

Figure S3: Hot-carrier cooling temperature as a function of delay time in $\mathrm{MAPbI}_{3}$ films at different pump fluences in response to a 370-nm excitation. The corresponding carrier densities are quoted in parentheses. 

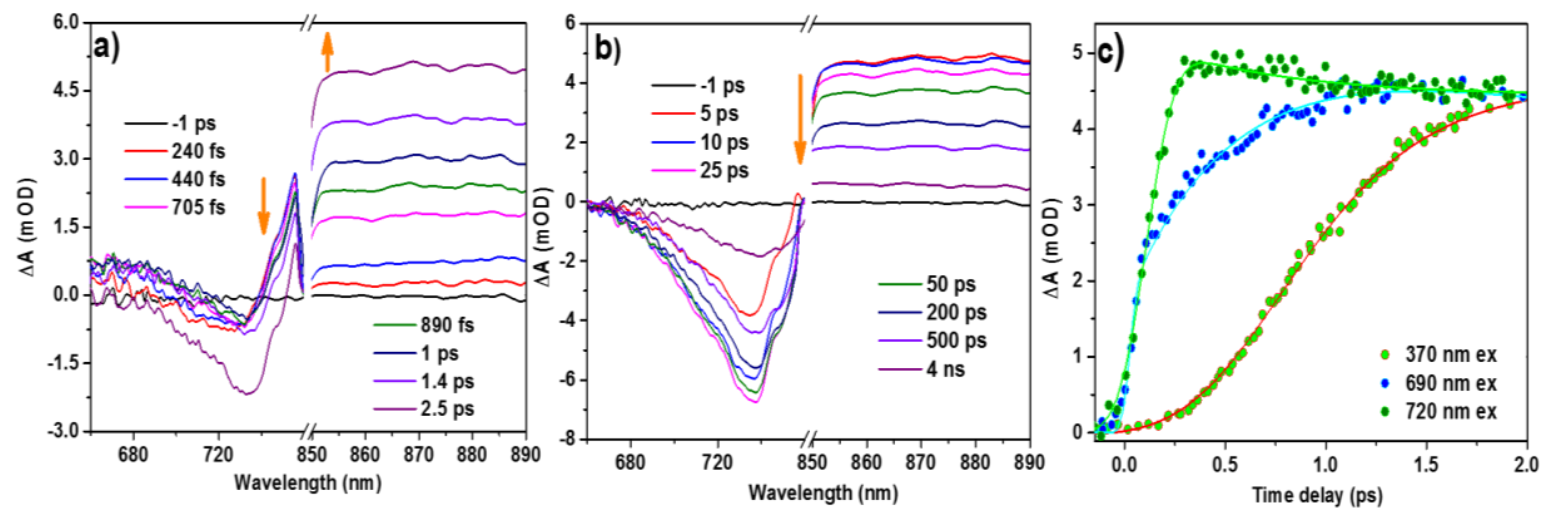

Figure S4: fs-TA spectra of $\mathrm{MAPbI}_{3}$ films at a) early and b) long time delays in response to a $370 \mathrm{~nm}$ optical excitation. c) The free carrier formation as a function of optical excitation. The solid lines stand for the fitting curves of the experimental data. Different pump excitations dependent measurements were performed at a fixed pump fluence $\left(\sim 3 \mu \mathrm{J} / \mathrm{cm}^{2}\right)$. 


\section{WILEY-VCH}
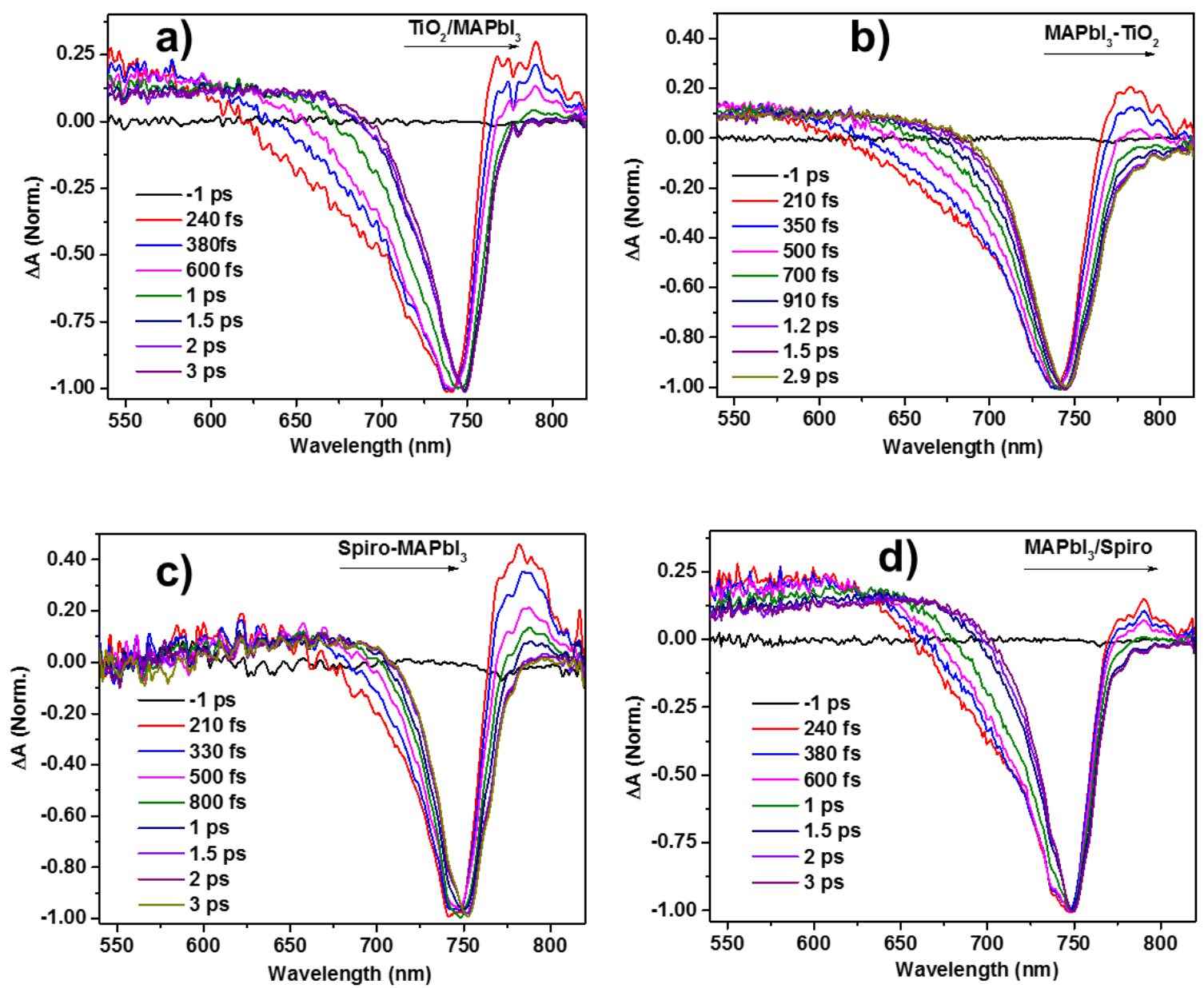

Figure S5: Normalized fs-TA spectra of $\mathrm{MAPbI}_{3}-\mathrm{TiO}_{2}$ (a), (b) and $\mathrm{MAPbI}_{3}-\mathrm{Spiro}$ (c), (d) in response to a 370-nm excitation (with a fixed pump fluence of $3 \mu \mathrm{J} / \mathrm{cm}^{2}$ ) for the extraction layer side and perovskite side, respectively (arrow shows the direction of the excitation). 


\section{WILEY-VCH}
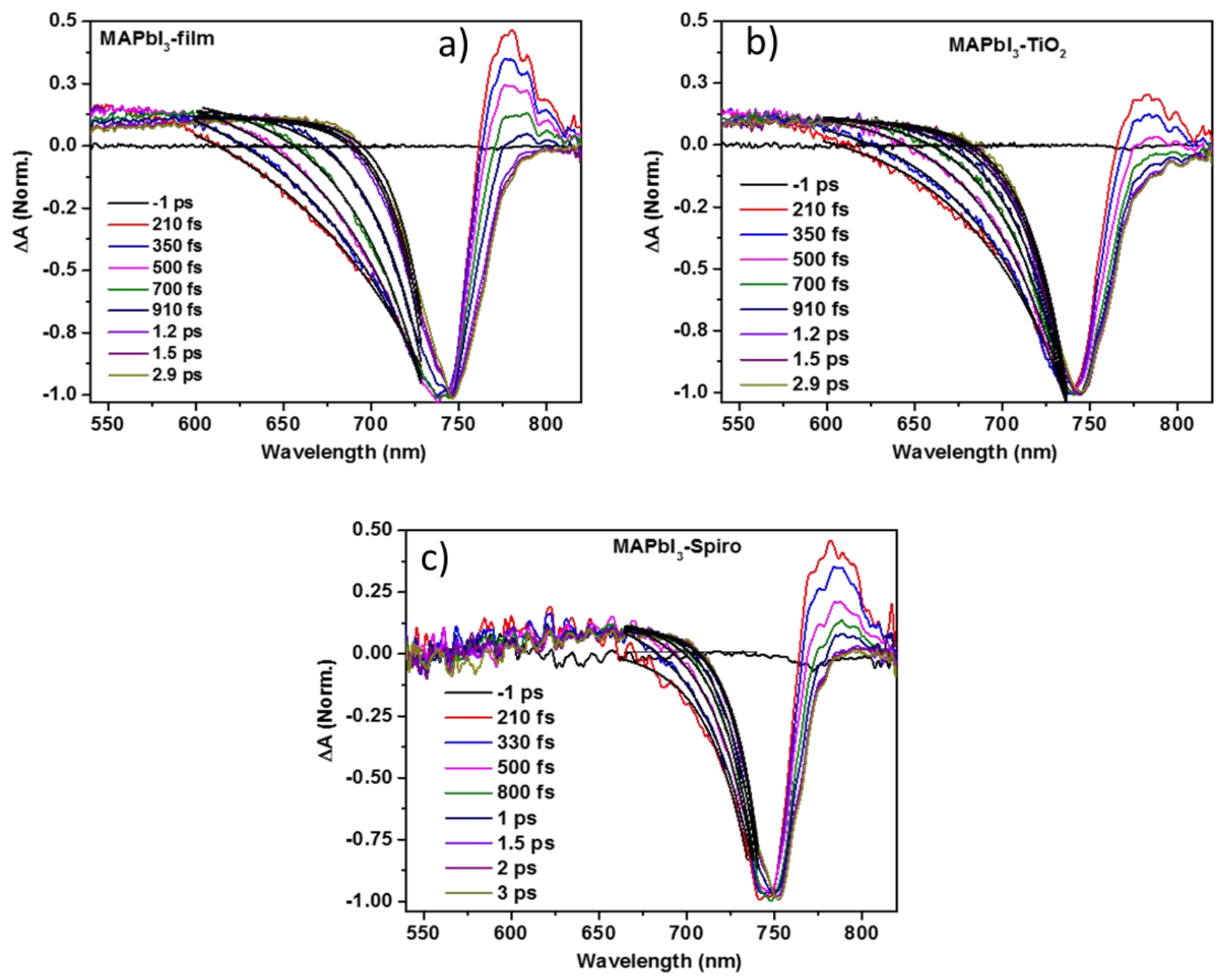

Figure S6: Single exponential fitting curves (black solid lines) of the high-energy tails in the normalized fs-TA spectra of a) $\mathrm{MAPbI}_{3}$ film, b) $\mathrm{MAPbI}_{3} / \mathrm{TiO}_{2}$, and c) $\mathrm{MAPbI}_{3} /$ Spiro in response of 370-nm optical pulses having a fixed pump fluence of $3 \mu \mathrm{J} / \mathrm{cm}^{2}$. 


\section{WILEY-VCH}
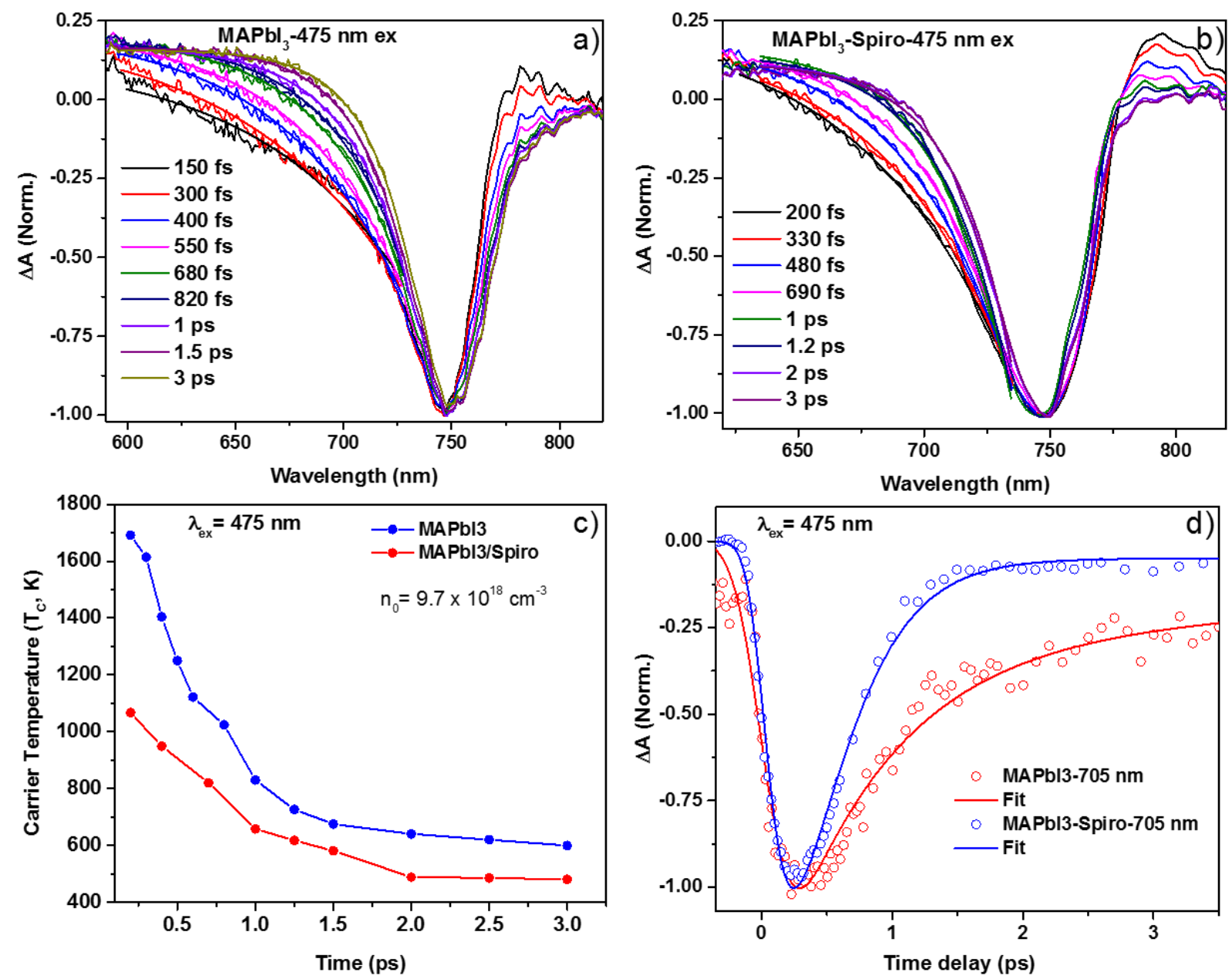

Figure S7: Normalized fs-TA spectra of the a) $\mathrm{MAPbI}_{3}$ film, b) $\mathrm{MAPbI}_{3} / \mathrm{Spiro-OMeTAD}$, in response to a 475-nm excitation with a fixed pump fluence of $\sim 3 \mu \mathrm{J} / \mathrm{cm}^{2}$. c) Hot carrier cooling as a function of delay time, extracted from a) and b). d) Normalized TA kinetics taken at 705 $\mathrm{nm}$. 


\section{WILEY-VCH}
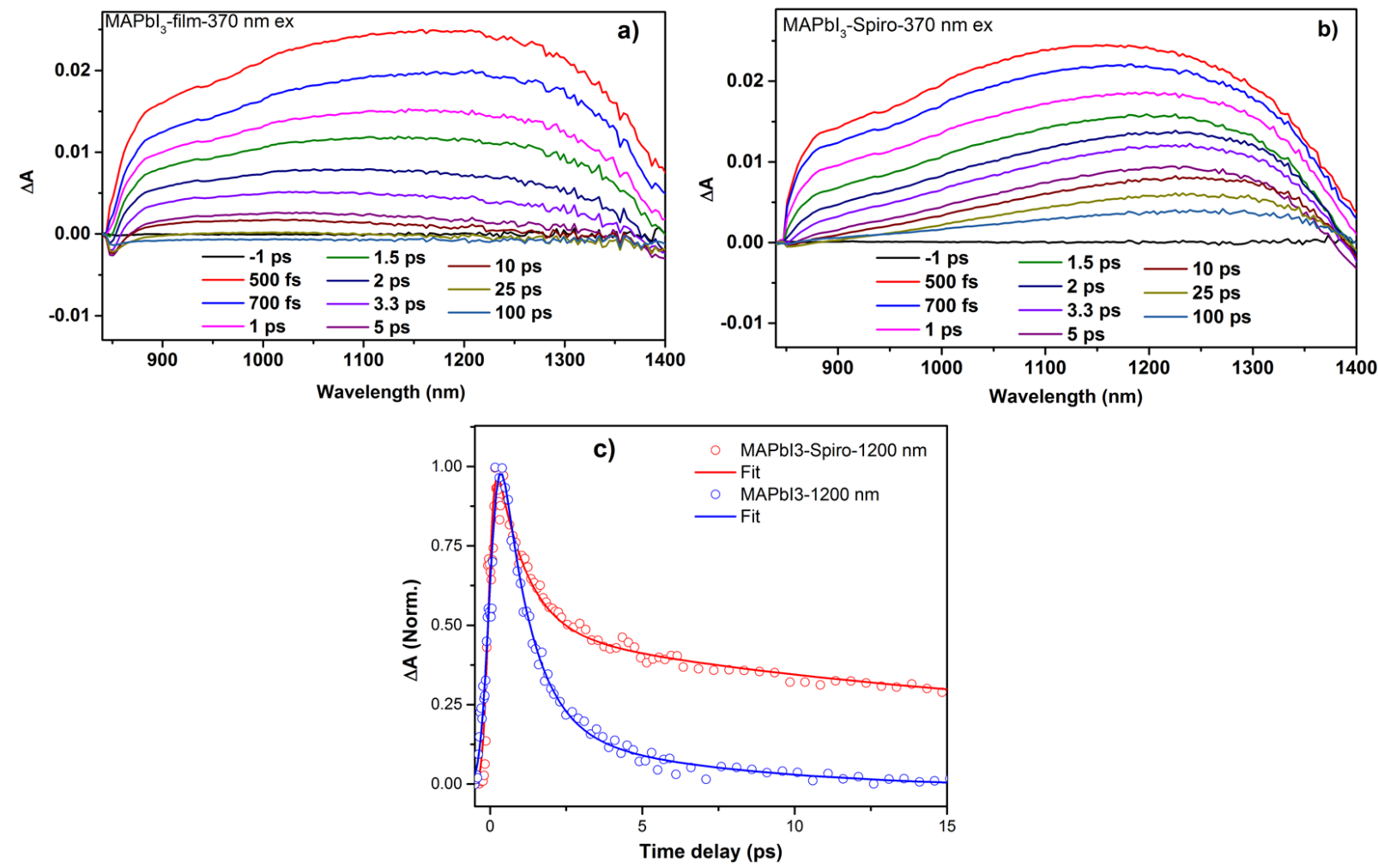

Figure S8: fs-TA spectra at different time delay in NIR region of $\mathrm{MAPbI}_{3}$ a) and $\mathrm{MAPbI}_{3}-$ Spiro-OMeTAD b) in response to 370-nm optical excitation having a fixed pump fluence of 3 $\mu \mathrm{J} / \mathrm{cm}^{2}$. c) Normalized fs-TA kinetic of $\mathrm{MAPbI}_{3}$ (blue) and $\mathrm{MAPbI}_{3}-\mathrm{Spiro}-\mathrm{OMeTAD}$ (red) at $1200 \mathrm{~nm}$. 


\section{WILEY-VCH}

Table S1: PL decay fitting parameters of $\mathrm{MAPbI}_{3}, \mathrm{MAPbI}_{3}$ with ETL and HTL following excitation at $650 \mathrm{~nm}$ laser pulse. The percentage at the parenthesis represents amplitude of the corresponding exponential functions.

\begin{tabular}{|c|c|c|c|}
\hline Systems & Average (ns) & $\mathbf{t}_{\mathbf{2}}(\mathbf{n s})$ & $\mathbf{t}_{\mathbf{2}}(\mathbf{n s})$ \\
\hline $\mathrm{MAPbI}_{3}$ & $2.76 \pm 0.12$ & $1.1(88 \%)$ & $14.9(12 \%)$ \\
\hline $\mathrm{TiO}_{2} / \mathrm{MAPbI}_{3}$ & $0.68 \pm 0.04$ & $0.55(97.5 \%)$ & $5.9(2.5 \%)$ \\
\hline $\mathrm{MAPbI}_{3} / \mathrm{Spiro}-\mathrm{OMeTAD}$ & $0.76 \pm 0.04$ & $0.6(97 \%)$ & $6(3 \%)$ \\
\hline
\end{tabular}

Supporting Information References

1- Y. Kanemitsu, J. Mater. Chem. C 2017, 5, 3427.

2- Richter 2017 J. M. Richter, F. Branchi, F. Valduga de Almeida Camargo, B. Zhao, R. H.

Friend, G. Cerullo, F. Deschler, Nat. Commun. 2017, 8, 376; 